\title{
A NOTE ON COMMUTATIVE BAER RINGS
}

\author{
T. P. SPEED \\ (Received 24 March 1970; revised 1 May 1970) \\ Communicated by B. Mond
}

In this note we study commutative Baer rings, uniting the abstract algebraic approach with the approach of [3] using minimal prime ideals. Some new characterisations of this class of rings are obtained, relations between the minimal prime ideals of a commutative Baer ring $B$ and its algebra $E_{B}$ of idempotents are considered, and some results concerning the direct decomposition of commutative Baer rings are given. We then study Baer ideals, and finally state without proof a new construction of the Baer extension of a commutative semiprime ring.

It is a pleasure to acknowledge valuable discussions with Michael Evans on many aspects of this topic, and with Don Taylor on the material in section 6.

\section{1}

Throughout our notation and terminology will be the same as in [3], apart from some slight changes. Proofs of most of the basic results concerning minimal prime ideals are given in [3] and we will use these without comment.

In this section $A$ denotes a commutative ring with identity, and we will give some necessary and sufficient conditions for $A$ to be a commutative Baer ring. $I(A)$ denotes the lattice of all ideals of $A$.

THEOREM 2.1. For a commutative semiprime ring $A$ satisfying the following condition: $C\left(^{*}\right)$ : for every $a \in A$ there exists $a^{\prime} \in A$ such that $(a)^{* *}=\left(a^{\prime}\right)^{*}$ the following are pairwise equivalent:

(i) $(a)^{*}+(a)^{* *}=A$ for every $a \in A$.

(ii) $(a b)^{*}=(a)^{*}+(b)^{*}$ for every $a$ and $b \in A$.

(iii) $\quad\left\{(a)^{* *}: a \in A\right\}$ is a Boolean sublattice of $I(A)$.

(iv) For every $a$ and $b \in A$ such that $a b=0$ there exists $e=e^{2}$ such that $a e=a$ and $b(1-e)=b$. 
Proor. $(i) \Rightarrow$ (ii). If $(a)^{*}$ and $(b)^{*}$ are direct summands of $A$ then there are idempotents $e$ and $f$ such that $(a)^{*}=(e),(b)^{*}=(f)$. From this we have

$$
\begin{aligned}
& \begin{aligned}
(a b)^{*} & =(a b)^{* * *}=\left((a)^{* *} \cap(b)^{* *}\right)^{*}=((1-e) \cap(1-f))^{*}=(e+f-e f) \\
& =(e)+(f)=(a)^{*}+(b)^{*} .
\end{aligned} \\
& \text { (ii) } \Rightarrow \text { (iii) Using condition } C\left(^{*}\right) \text { we see that }
\end{aligned}
$$

$$
(a)^{* *}+(b)^{* *}=\left(a^{\prime}\right)^{*}+\left(b^{\prime}\right)^{*}=\left(a^{\prime} b^{\prime}\right)^{*}=\left(\left(a^{\prime} b^{\prime}\right)^{\prime}\right)^{* *}
$$

also $(a)^{* *} \cap(b)^{* *}=(a b)^{* *}$ is always valid.

(iii) $\Rightarrow$ (iv) and (iv) $\Rightarrow$ (i) are easy to prove and we omit the details.

Our next result shows that under a suitable extra hypothesis, a converse to a result of Kist can be obtained.

THEOREM 2.2. Let $A$ be a commutative semiprime ring satisfying the following condition $G C\left(^{*}\right)$ : for every $a \in A$ there exists $\left\{a_{1}, \cdots, a_{m}\right\} \subseteq A$ such that $(a)^{* *}=\bigcap_{i=1}^{m}\left(a_{i}\right)^{*}$. Then $A$ is a commutative Baer ring iff for every pair of distinct minimal prime ideals $M$ and $N$ of $A$ we have $M+N=A$.

PROof. The direct part of the theorem is Theorem 9.5 of [3]. For the converse suppose that A satisfies $G C\left(^{*}\right)$ but there exists $a \in A$ with $(a)^{*}+(a)^{* *} \neq A$. Then we can find a (proper) prime ideal $P$ of $A$ with $(a)^{*}+(a)^{* *} \subseteq P \subseteq A$. By our assumption $P$ contains a unique minimal prime ideal and so the localisation at $P$, the ring $A_{P}$, contains a unique minimal prime, i.e. is an integral domain. If $x \rightarrow x / 1$ is the canonical map from $A$ into $A_{P}$ then $a / 1 \neq 0 / 1$ since $P \supseteq(a)^{*}$. Also if $a_{i} / 1=0 / 1$ for every $i$ we would have $a_{i} s_{i}=0$ for some $s_{i} \notin P(1 \leqq i \leqq m)$ whence $s=s_{1} \cdot s_{2} \cdots \cdot s_{m} \notin P$ satisfies

$$
s \in \bigcap_{i=1}^{m}\left(a_{i}\right)^{*}=(a)^{* *} \subseteq P,
$$

an impossibility. So $a_{j} / 1 \neq 0 / 1$ for some $j$. But now we obtain the final contradiction, for $a / 1 \cdot a_{j} / 1=0 / 1$ which is impossible in the integral domain $A_{l}$. Hence $(a)^{*}+(a)^{* *}=A$ for every $a \in A$, and the result is proved

Remark. The extra hypothesis $G C\left(^{*}\right)$ is necessary. For if $X$ is a compact $F$-space which is not basically disconnected, the ring $C(X)$ is not Baer, but distinct minimal primes are comaximal. For details see [2].

We now exhibit some connections between the minimal primes of a commutative Baer ring $B$ and the Boolean lattice $E_{B}$ of idempotents of $B$. The following lemmas are easily established. 
LeMmA 3.1. In any commutative Baer ring $B$, if $a b=0$ then $(a+b)^{*}$ $=a^{*} b^{*}$ and $(a+b)^{* *}=a^{* *}+b^{* *}$.

LEMMA 3.2. In any commutative ring $A$, if $a \in A$ can be written as $a=\sum_{i=1}^{m} a_{i} e_{i}$ where $e_{i}^{2}=e_{i}(1 \leqq i \leqq m)$ then there are coefficients $b_{i}$ and orthogonal idempotents $f_{i}(1 \leqq i \leqq m)$ with $f_{i} \leqq e_{i}(1 \leqq i \leqq m)$ and $a=\sum_{i=1}^{m} \quad b_{i} f_{i}$.

THEOREM 3.3. Let $A$ be a commutative ring with identity. If $M$ is a prime ideal of $A$ then $M \cap E_{A}$ is a prime ideal of $E_{A}$. Conversely, if $P$ is a prime ideal of $E_{A}$, and $A$ is a commutative Baer ring, then the ideal $(P)$ of $A$ generated by $P$ is a minimal prime ideal of $A$.

Proof. The first statement is straightforward. We will show that $(P)$ is a minimal prime of $A$ if $P$ is a prime of $E_{A}$. Suppose $a b \in(P)$, i.e. $a b=\sum_{i=1}^{m} x_{i} e_{i}$ for $x_{i} \in A$, and $e_{i} \in P$ with $1 \leqq i \leqq m$. Then by 3.2 we also have $a b=\sum_{i=1}^{m} y_{i} f_{i}$ where $y_{i} \in A$ with $1 \leqq i \leqq m$ and the $\left\{f_{l}\right\}$ is a set of orthogonal idempotents, also in $P$. By a generalized form of 3.1 we have

$$
a^{* *} b^{* *}=(a b)^{* *}=\sum_{i=1}^{m}\left(y_{i} f_{i}\right)^{* *}=\sum_{i=1}^{m} y_{i}^{* *} f_{i} \in P
$$

whence $a^{* *} \in P$ or $b^{* *} \in P$. This immediately implies that $a \in(P)$ or $b \in(P)$, and so $(P)$ is prime. Finally $(P)$ is minimal prime since $a=\sum_{i=1}^{m} a_{i} e_{i} \in(P)$ for $\left\{e_{i}\right\} \subseteq P$ implies that

$$
g=\left(1-e_{1}\right)\left(1-e_{2}\right) \cdots\left(1-e_{m}\right) \notin P
$$

and so $g \notin(P)$, and $a g=0$. The proof is complete.

This result enables us to obtain another characterisation of commutative Baer rings.

THEOREM 3.4. Let $A$ be a commutative semiprime ring satisfying $G C\left(^{*}\right)$ of 2.2. Then $A$ is a commutative Baer ring iff for any minimal prime ideal $M$ of $A$ we have $M=\left(M \cap E_{A}\right)$.

Proof. It is straightforward to prove using 3.3 that if $A$ is a commutative Baer ring then $M=\left(M \cap E_{A}\right)$ for $M$ minimal prime. For the converse, suppose $M$ and $N$ are distinct minimal primes of $A$. By hypothesis we must have $M \cap E_{A}$ and $N \cap E_{A}$ distinct prime, and hence maximal, ideals of $E_{A}$. Thus $M \cap E_{A}$ and $N \cap E_{A}$ together generate $E_{A}$ and so $M+N=\mathrm{A}$. The theorem now follows from 2.2.

\section{4}

It is easy to show that (arbitrary) direct products of integral domains are commutative Baer rings. We now consider possible converse results. 
LEMMA 4.1. Let $B$ a commutative Baer ring and $e$ an atom of the Boolean algebra $E_{B}$ of idempotents. Then $(e)_{B}$ is an integral domain.

Proof. Suppose $a b=0$ for $a$ and $b$ in $(e)_{B}$. Then $a^{* *} b^{* *}=0$ and, since $e$ is an atom, $a^{* *}=0$ or $e$ and $b^{* *}=0$ or $e$. Clearly either $a^{* *}$ or $b^{* *}$ must be 0 whence either $a$ or $b$ is zero.

THEOREM 4.2. Let $B$ be a commutative Baer ring. Then $B$ is Noetherian iff $B$ is a finite direct product of Noetherian integral domains. If $B$ is finite then it is a finite product of finite fields.

Procf. Suppose $B$ is a Noetherian commutative Baer ring. Then $B$ possesses only finitely many minimal prime ideals and so we deduce by 3.4 that $E_{B}$ is finite, hence atomic. If the atoms of $E_{B}$ are $e_{1}, \cdots, e_{m}$, then it is easy to check that the map $a \rightarrow\left\langle a e_{i}\right\rangle_{i=1}^{n}$ for $a \in B$ defines as isomorphism of $B$ onto the product $\chi_{i=1}^{m}\left(e_{i}\right)_{B}$ of domains, each of which is clearly Noetherian. The converse and the final remark are both clear.

Next we ask when a commutative Baer ring $B$ is a product of possibly infinitely many integral domains. To do this we use the partial order $\leqq$ on any commutative semiprime ring $A$ which extends that on $E_{A}$, given by $a \leqq b$ if $a b=a^{2}$. For details and some results which we shall use, see [1]. A subset $S \subseteq A$ is said to be orthogonal if $s t=0$ for $s, t \in S$ with $s \neq t$. The ring $A$ is said to be orthogonally complete if every orthogonal subset has a join relative to the partial order $\leqq$.

THEOREM 4.3. Let the ring $A$ be isomorphic to a direct product of integral domains. Then $A$ is a commutative Baer ring satisfying:

(i) $E_{A}$ is an atomic Boolean ring;

(ii) $A$ is orthogonally complete.

Conversely, let $A$ be a commutative Baer ring satisfying (i) and (ii) above. Then $A$ is isomorphic to a direct product of integral domains.

Proof. Let $\phi: A \rightarrow \chi_{i \in I} D_{i}$ be an isomorphism of $A$ onto a product of domains, such that $a \phi=\left\langle a_{i}\right\rangle_{i \in I}$. Then by results in [1] we see that $A$ is a commutative Baer ring, and the idempotents of $A$ are the inverse images of element $\left\langle e_{i}\right\rangle_{i \in I}$ where $e_{i}=0_{i}$ with $i \in I_{1}$ and $e_{i}=1_{i}$ with $i \in I \backslash I_{1}$, for $I_{1}$ any subset of $I$. Thus $E_{A}$ is seen to be isomorphic to the Boolean ring of subsets of $I$, which is atomic. The fact that $A$ is orthogonally complete is proved exactly as in [1] p. 506 and so we omit the details.

Now for the converse. Index the atoms of $E_{A}$ by the set $I$ and for the atom $e_{i}$ write $D_{i}=\left(e_{i}\right)_{A}$, a domain by 4.1 . We define a map

$$
\psi: A \rightarrow \underset{i \in I}{X}\left(e_{i}\right) \text { by } a \psi=\left\langle a e_{i}\right\rangle_{i \in I} .
$$


It is easy to see that the elements $a e_{i}$ are all zero if $a=a^{* *}=0$, and so $\psi$ is seen to be a ring monomorphism. Suppose $\left\langle a_{i}\right\rangle \in X_{i \in I}\left(e_{i}\right)$, then the set $\left\{a_{i}: i \in I\right\}$ is orthogonal in $A$ and hence has a supremum $a$ (relative to $\leqq$ ). Then by a result in [1] we have

$$
a e_{j}=\left(\underset{i}{\bigvee} a_{i}\right) e_{j}=\bigvee_{i} a_{i} e_{j}=a_{j}
$$

and so $a \psi=\left\langle a_{i}\right\rangle_{i \in I}$ proving that $\psi$ is onto, and completing our proof.

REMARK. In a first draft of this work an equivalent theorem was proved which did not use the partial order; after seeing [1] the above neater formulation was obtained.

Next, we consider Baer-ideals, the kernels of the morphisms of a commutative Baer ring when the latter is considered as an abstract algebra.

THEOREM 5.1. Let $B$ be a commutative Baer ring. Then the following are pairwise equivalent, for an ideal $J$ of $B$.

(i) $J$ is a Baer-ideal, i.e., $a-b \in J$ implies $a^{*}-b^{*} \in J$.

(ii) If $a \in J$ and $a^{*}=b^{*}$ then $b \in J$.

(iii) $a \in J$ iff $a^{* *} \in J$.

(iv) $J=\cap\left\{M \in \mathscr{M}_{B}: M \supseteq J\right\}$.

Here $\mathscr{M}_{B}$ is the set of all minimal prime ideals of $B$.

Proof. (i) $\Rightarrow$ (ii) Suppose $J$ is a Baer-ideal, and $a \in J$ and $b^{*}=a^{*}$. Then $a^{*}-1=b^{*}-1 \in J$ and so $b^{* *} \in J$ whence $b=b b^{* *} \in J$, proving (ii).

(ii) $\Rightarrow$ (iii) If $a \in J$ then $\left(a^{* *}\right)^{*}=a^{*}$ implies $a^{* *} \in J$. Also $a^{* *} \in J$ implies $a=a^{* *} a \in J$.

(iii) $\Rightarrow$ (iv) We note firstly that $J$ is a radical ideal. For if $a^{n} \in J$ then $\left(a^{n}\right)^{* *}=a^{* *} \in J$ and so $a=a a^{* *} \in J$. This means that $J$ is the intersection of all the prime ideals of $B$ minimal with respect to containing $J$, and we now show that each such ideal is actually a minimal prime ideal of $B$. Let $P$ be a minimal prime belonging to $J$; there exists for every $a \in P$ an element $x \in P$ such that $a x \in J$. Clearly $(a x)^{* *} \subseteq J \subseteq P$ and so $(a x)^{*} \nsubseteq P$ since $B$ is a Baer ring. Thus there is $t \in(a x)^{*} \mid P$ i.e. $t \notin P$ such that $a x t=0$. But $x t \notin P$ and hence $P$ is characterised as a minimal prime ideal of $B$, and $J$ is the intersection of minimal primes as asserted.

(iv) $\Rightarrow$ (i) We omit the easy proof that any minimal prime ideal, and hence any intersection of minimal primes, is a Baer idael.

Corollary 5.2. For any $K \subset B, K^{*}$ is a Baer-ideal. 
PROOF. $K^{*}=\cap\left\{M \in \mathscr{M}_{B}: M \nsubseteq K\right\}$ by a result in [3].

THEOREM 5.3 Let $B$ be a commutative Baer ring. Then the lattice $I_{b}(B)$ of all Baer-ideals of $B$ is isomorphic to the lattice $I\left(E_{B}\right)$ of all ideals of the Boolean ring $E_{B}$.

Proof. The map $J \leftrightarrow J \cap E_{B}$ of $I_{b}(B)$ into $I\left(E_{B}\right)$ is mono by 5.1 (iii) above. It is certainly onto all prime ideals of $E_{B}$ by 3.4 and so, using 5.1 (iv) it is onto $I\left(E_{B}\right)$. Since the map is clearly order-preserving the theorem is proved.

COROLlary 5.4. The lattice $I_{b}(B)$ of all Baer-ideals of $B$ is a complete, relatively pseudo-complemented (and hence distributive) lattice. $I_{b}(B)$ is a Stone lattice if $E_{B}$ is a complete Boolean algebra.

Proof. These facts follow from known results concerning the lattice of ideals of a Boolean algebra and 5.3.

To close this note we mention an alternative construction of the Baer extension of a commutative semiprime ring. The main virtue of our approach is that it enables (i) a functorial characterisation of the map $A \rightarrow B(A)$ and (ii) a characterisation of the map $A \rightarrow B(A)$ in terms of the dual semilattice of [3], to be given. We do not give any details as the proofs are all very similar to ones appearing in another context [4]. Let us call a ring morphism $\phi: A \rightarrow A^{\prime} R$-compatible if $(a)^{*}=(b)^{*}$ in $A$ implies $(a \phi)^{*}=(b \phi)^{*}$ in $A^{\prime}$. It is easy to see that if $A$ and $A^{\prime}$ are commutative Baer rings then $\phi$ is $R$-compatible if the kernel $\operatorname{ker} \phi$ of $\phi$ is a Baer-ideal of $A$.

THEOREM 6.1. Let $A$ be a commutative semiprime ring. Then there is a commutative Baer ring $B(A)$ and an $R$-compatible ring monomorphism $\beta: A \rightarrow B(A)$ with the following property: for every $R$-compatible ring morphism $\phi: A \rightarrow B$ of $A$ into a commutative Baer ring $B$, there is a unique Baer morphism $\phi: B(A) \rightarrow B$ such that $\beta \circ \phi=\phi$. The pair $(\beta, B(A))$ is unique.

COROLlaRY 6.2. Let $\mathrm{E}$ be a commutative Baer ring. Then there is a Baer monomorphism $\beta$ from $B$ onto a Baer subring of a direct product of integral domains.

For the next corollary we need some notation. If $\mathscr{M}_{A}$ is the set of minimal prime ideals of $A$, the dual semilattice is

$$
\mu_{A}=\left\{\mathscr{M}_{A}(a): a \in A\right\}
$$

where $\mathscr{M}_{A}(a)=\left\{M \in \mathscr{M}_{A}: a \notin M\right\}$. We write $\bar{\mu}_{A}$ for the Boolean lattice generated by $\mu_{A}$. If $\phi: A \rightarrow A^{\prime}$ is $R$-compatible, we have an induced map 


$$
\phi^{*}: \mu_{A} \rightarrow \mu_{A}^{\prime} \text { given by } \mathscr{M}_{A}(a) \phi^{*}=\mathscr{M}_{A^{\prime}}(a \phi),
$$

and this map extends to $\bar{\mu}_{A}$.

Corollary 6.3. The pair $(\beta, B(A))$ satisfies the following conditions:

(i) $\beta: A \rightarrow B(A)$ is an $R$-compatible ring monomorphism of $A$ into $a$ commutative Baer ring;

(ii) The induced map $\beta^{*}: \bar{\mu}_{A} \rightarrow \mu_{B(A)}$ is a Boolean isomorphism;

(iii) For every $s \in B(A)$ there are elements $a_{1}, \cdots, a_{m}$ of $A$ and idempotents $e_{1}, \cdots, e_{m}$ of $B(A)$ with $e_{i} e_{j}=0(i \neq j)$ and $\sum_{i=1}^{m} e_{i}=1$ such that $s=\sum_{i=1}^{m}\left(a_{i} \beta\right) e_{i}$.

Thus our Baer extension satisfies conditions given in Kist [3]. We now give our final result.

THEOREM 6.4. Let $(k, K(A))$ be an extension of the commutative semiprime ring $A$ satisfying conditions (i), (ii), (iii) of 6.3. Then there are Baer isomorphisms $\bar{k}: B(A) \rightarrow K(A)$ and $\bar{\beta}: K(A) \rightarrow B(A)$ such that $\bar{k} \circ \bar{\beta}={ }_{B(A)}{ }_{B(B} \circ \bar{k}={ }_{K(A)}{ }^{\prime}$.

\section{References}

[1] A. Abian, 'Direct Product Decomposition of Commutative Semisimple Rings', Proc. Amer. Math. Soc. 24 (1970), 502-507.

[2] L. Gillman and M, Jerison, Rings of Continuous Functions (Van Nostrand 1960.)

[3] J. Kist, 'Minimal Prime Ideals in Commutative Semigroups', Proc. Lond. Math. Soc. Ser.3, 13 (1963), 31-50.

[4] T. P. Speed, 'A Note on Stone Lattices', Can. Math. Bull. 14 (1971) 81-86.

Department of Probability \& Statistics

The University

Sheffield, S3 7RH

England 